\title{
To Be Screened or Not to Be...? Psyche First?
}

\author{
Jaroslaw Regula ${ }^{1,2}$
}

1) Department of

Gastroenterology, Hepatology and Clinical Oncology, Center of Postgraduate Medical Education, Warsaw;

2) Department of Gastroenterological Oncology, The Maria Sklodowska-Curie National Research Institute of Oncology, Warsaw, Poland

\footnotetext{
Address for correspondence: Jaroslaw Regula

Department of

Gastroenterology, Hepatology and Clinical Oncology, Center of Postgraduate Medical Education,

Warsaw, Poland

Jaroslaw.Regula@pib-nio.pl
}

Received: 14.09.2020 Accepted: 01.10.2020
Population adherence to cancer screening recommendations is not satisfactory in many countries. Public Health organizations have struggled to find ways to improve the situation. Research is ongoing; however, no ideal methodology has been found, so far. In this issue of the Journal of Gastrointestinal and Liver Diseases two very interesting and valuable research studies on this topic are presented $[1,2]$.

First, a study performed in Romania on a sample of people living in an urban area aged 50 years or more attending day care centers for elderly [1]. Of those, almost a quarter (26\%) reported having previous colonoscopy screening despite the fact that Romania at the time of the study had no screening programme introduced yet. The authors intended to better understand psychological factors behind the positive decisions of undergoing screening and compare to those who were not screened and prepared questionnaires in order to compare the psychological factors between screeners and non-screeners. Formal validated tests were used assessing the Health Literacy, Health Belief Model (including perceived barriers and perceived benefits) and Self-efficacy surveys. The authors also included dimensions related to the organization of the healthcare system. They concluded that the main discriminators between the groups were: the ability to perceive benefits of screening, knowledge of previous recommendations for screening and knowledge of previous recommendations for prevention. Of those, the most important factor was the ability to perceive the benefits of screening. Interestingly in this cohort, the knowledge of perceived barriers was not discriminatory. This is in accordance with other publications stating that perceived benefits weigh more than perceived barriers when taking a decision to attend a colonoscopy screening or not. This is very interesting and positive information meaning that health providers organizing colorectal cancer screening should provide balanced and honest information, mainly on the benefits but of course also presenting barriers (the harms) for potential screeners to decide which is more important. Recently, a valuable study showed that presenting the benefits and the barriers of different screening methods can be made in a user friendly graphical way for lay people to easier understand differences [3]. Benefits were presented as the numbers of avoided cancers and numbers of avoided cancer death per 1,000 people who participated in the screening. Such methods could be used to provide comprehensible information for potential screeners. In this study, the numbers of colonoscopies which were required to be performed were used as a measure of the barriers. Another method of presenting the harm factor could be also mentioning the risk of interval cancers. These are cancers that were diagnosed between the screening episodes and in fact are a measure of failed screening (cancers missed by screening). This aspect was shown in the recent Italian study presenting the rate of interval cancers following 7 rounds of a faecal immunochemical test (FIT) screening programme [4]. Authors drew the graph with dots representing as many as 10,000 individuals who underwent FIT screening with colonoscopy for positive results. The overwhelming majority of the dots were green representing negative screening tests and only a single red dot indicated interval cancers, which is very encouraging and may strengthen the decisions of potential screeners.

A second study in the current issue of the Journal of Gastrointestinal and Liver Diseases on the topic, offered the answer to a question whether knowledge of positive cancer family history may influence the decision to undergo colorectal cancer screening [2]. Naturally, the intuitive answer is "yes”. However, available research so far, has given mixed results. The authors completed a study within the randomized controlled 
trial of Helicobacter pylori eradication and pepsinogen testing for the prevention of gastric cancer mortality (GISTAR study) performed in four centers in Latvia. Extensive questionnaires were distributed among 3,239 participants of the study containing self-reported data on cancer family history, as well as sociodemographic data, medical investigations in the last three years and modifiable cancer risk factors (smoking, dietary habits, alcohol consumption, body mass index). The principal analysis contained the comparison between those who had positive family history versus those with negative family history. There were $56 \%$ of participants with a family history of cancer. It turned out that participants with a positive family history were significantly more likely to report a history of gastrointestinal investigations, especially colorectal cancer screening in the last three years (fecal occult blood tests, colonoscopies). The knowledge of a positive family history was also associated with less frequent harmful risk habits (smoking and alcohol consumption). Such a powerful effect! The authors rightly concluded that the knowledge of a positive family history may lead to the modification of lifestyle and medical behaviors. If so, why not use this actively to increase participation rates in screening activities by searching out such people and educating them.

However, one must state that this should be used cautiously. First, the quality of family history details may be variable in different societies. Also, knowledge about family history is different between men and women. Finally, paternity may not mean always biological links and therefore information on family history may be misleading in an undefined fraction of people. Therefore, family history has been rather underused and poorly understood, so far.

Both studies presented in the current issue of the journal are very interesting, showing that the understanding of detailed psychological analysis of human behaviors and motivations including also the role of family history knowledge may help increasing compliance to colorectal cancer screening. This would require the modification of educational materials and the promotion of screening by healthcare professionals. Most probably a single factor is not powerful enough to increase compliance sufficiently. However, recent data indicate that combining high quality family history with genetic testing may lead to better quantification of the colorectal cancer risk [5] and be more specific in promotional activities.

We need to be better prepared in future to answer the modified Shakespeare's question in this Editorial's title and to develop the use of more sophisticated arguments to be sure of success.

Conflicts of interest: None to declare.

\section{REFERENCES}

1. Ciuca A, Moldovan R, Pintea S, Dumitrascu D, Baban A. Screeners vs non-screeners for colorectal cancer among people over 50 years of age: factual and psychological discriminants. J Gastrointestin Liver Dis 2020;29:529-537. doi:10.15403/jgld-2263

2. Razuka-Ebela D, Zile I, Tzivian L, et al. J Does Family History of Cancer Influence Undergoing Screening and Gastrointestinal Investigations? J Gastrointestin Liver Dis 2020;29:523-528. doi:10.15403/jgld-813.

3. Helsingen LM, Vandvik PO, Jodal HC, et al. Colorectal cancer screening with faecal immunochemical testing, sigmoidoscopy or colonoscopy: a clinical practice guideline. BMJ 2019;367:15515. doi:10.1136/bmj.15515

4. Zorzi M, Hassan C, Senore C, et al. Interval colorectal cancers after negative faecal immunochemical test in a 13-year screening programme. J Med Screen 2020 May 11. doi:10.1177/0969141320918613

5. Kastrinos F, Samadder NJ, Burt RW. Use of Family History and Genetic Testing to Determine Risk of Colorectal Cancer. Gastroenterology 2020;158:389-403. doi:10.1053/j.gastro.2019.11.029 